Supporting information

\title{
Zeolitic Tetrazolate-Imidazolate Frameworks (ZTIFs) with High Chemical Stability for Selective Separation of Small Hydrocarbons
}

\author{
Min-Yu Li, Fei Wang* and Jian Zhang \\ State Key Laboratory of Structural Chemistry, Fujian Institute of Research on the Structure of \\ Matter, the Chinese Academy of Sciences, Fuzhou, Fujian 350002, P. R. China. E-mail: \\ wangfei04@fjirsm.ac.cn
}

\section{Materials and general methods}

All chemicals were obtained from commercial sources and used without further purification. Elemental analyses of $\mathrm{C}, \mathrm{H}$ and $\mathrm{N}$ were measured on a Vario MICRO E III elemental analyzer. The IR spectra ( $\mathrm{KBr}$ pellets) were recorded on a Magna 750 FT-IR spectrophotometer. Powder X-ray diffraction data were recorded on a Rigaku MultiFlex diffractometer with a scan speed of $5^{\circ}$ $\min ^{-1}$. Thermal stability studies were carried out on a NETSCHZ STA-449C thermoanalyzer under $\mathrm{N}_{2}\left(30-600{ }^{\circ} \mathrm{C}\right.$ range) at a heating rate of $10{ }^{\circ} \mathrm{C} \mathrm{min}^{-1}$. Fluorescence spectra were measured with a HORIBA Jobin-Yvon FluoroMax-4 spectrometer. The gases adsorption isotherms were measured by using ASAP-2020 volumetric adsorption equipment.

Synthesis of $\mathbf{Z n}_{\mathbf{2}}(\mathbf{5}-\mathbf{m t z})_{\mathbf{3}}$ (2-mbim) $\cdot$ guest (ZTIF-6): the mixture of $\mathrm{Zn}\left(\mathrm{CH}_{3} \mathrm{CO}_{2}\right)_{2} \cdot 2\left(\mathrm{H}_{2} \mathrm{O}\right)(0.500$ mmol, $0.110 \mathrm{~g}$ ), 5-methyltetrazole (5-mtz, $0.500 \mathrm{mmol}, 0.042 \mathrm{~g}$ ), 2-methylbenzimdazole (2-mbim, $0.500 \mathrm{mmol}, 0.066 \mathrm{~g})$ in DMF $(2 \mathrm{~mL})$ and ethanol $(2 \mathrm{~mL})$ was sealed in a $20 \mathrm{~mL}$ vial and heated to $120{ }^{\circ} \mathrm{C}$ for 2 days, and then cooled to room-temperature. The pale yellow polyhedral crystals were obtained, washed with ethanol, and dried at room temperature (Yield: 60\%). Anal. Calcd for $\mathrm{C}_{14} \mathrm{H}_{16} \mathrm{~N}_{14} \mathrm{Zn}_{2}$ (activated): Calcd. C, 32.88; H, 3.13; N, 38.36; Found C, 33.02; H, 3.07; N, 38.48. IR ( $\left.\mathrm{KBr} \mathrm{cm}^{-1}\right): 2936$ (m), 1671 (s), 1499 (s), 1390 (vs), 1281 (m), 1095 (s), 760 (m), 695 (m).

Synthesis of $\mathbf{Z n}_{\mathbf{2}}(\mathbf{t z})_{\mathbf{2}}(\mathbf{2} \text {-mbim) })_{2} \cdot \mathbf{D M F}$ (ZTIF-7): the mixture of $\mathrm{Zn}\left(\mathrm{CH}_{3} \mathrm{CO}_{2}\right)_{2} \cdot 2\left(\mathrm{H}_{2} \mathrm{O}\right)(0.500$ 
mmol, $0.110 \mathrm{~g})$, tetrazole (tz, $0.500 \mathrm{mmol}, 0.035 \mathrm{~g}), 2$-methylbenzimdazole (2-mbim, $0.500 \mathrm{mmol}$, $0.066 \mathrm{~g})$ in DMF $(2 \mathrm{~mL})$ and ethanol $(2 \mathrm{~mL})$ was sealed in a $20 \mathrm{~mL}$ vial and heated to $120{ }^{\circ} \mathrm{C}$ for 2 days, and then cooled to room-temperature. The colorless polyhedral crystals were obtained, washed with ethanol, and dried at room temperature (Yield: $65 \%$ ). Anal. Calcd for $\mathrm{C}_{18} \mathrm{H}_{16} \mathrm{~N}_{12} \mathrm{Zn}_{2}$ (activated): Calcd. C, 40.66; H, 3.01; N, 31.63; Found C, 40.52; H, 3.17; N, 31.71. IR ( $\left.\mathrm{KBr} \mathrm{cm}^{-1}\right)$ : $2922(\mathrm{w}), 1669$ (s), 1457 (s), 1390 (vs), 1281 (m), 1075 (s), 758 (m), 687 (m).

Crystal data for ZTIF-6: Trigonal, $a=b=25.1117(9) \AA, c=13.2033(8) \AA, V=7210.5(8) \AA^{3}, T=$ 293(10) K, space group $R 3 m, Z=18,5412$ reflections measured, 1991 independent reflections $\left(R_{\text {int }}=0.0625\right)$. The final $R_{l}$ value was $0.0522(I>2 \sigma(I))$. The final $w R\left(F^{2}\right)$ value was $0.1412(I>$ $2 \sigma(I))$. The goodness of fit on $F^{2}$ was 1.012 .

Crystal data for ZTIF-7: Orthorhombic, $a=16.2940(4) \AA, b=16.6579(4) \AA, c=19.1723(4) \AA, V$ $=5203.8(2) \AA^{3}, T=293(2) \mathrm{K}$, space group $P b c a, Z=8,12189$ reflections measured, 4759 independent reflections $\left(R_{\text {int }}=0.0386\right)$. The final $R_{l}$ value was $0.0400(I>2 \sigma(I))$. The final $w R\left(F^{2}\right)$ value was $0.1036(I>2 \sigma(I))$. The goodness of fit on $F^{2}$ was 1.057 .

Table S1. Lower-Pressure $\mathrm{CO}_{2}$ Adsorption Capacities for SOD-type MOFs at 273K.

\begin{tabular}{|c|c|c|c|c|c|}
\hline Sample & Surfa & $\mathrm{a}\left(\mathrm{m}^{2} / \mathrm{g}\right)$ & Uptake $\left(\mathrm{cm}^{3} / \mathrm{g}\right)$ & $Q \mathrm{st}(\mathrm{kJ} / \mathrm{mol})$ & Ref. \\
\hline & BET & Langmuir & & & \\
\hline MAF-4 & & 1873 & 29.3 & $14.9-17.2$ & Adv. Mater. 2011, 23, 1268-1271 \\
\hline ZTIF-6 & 790 & 1142 & 53 & $18.6-19.8$ & This work \\
\hline MAF-7 & & 1874 & 62.5 & $23.8-25.1$ & Adv. Mater. 2011, 23, 1268-1271 \\
\hline TTF-4 & 1172 & 1692 & 67.6 & 16.7 & Chem. Commun. 2016, 52, 5625-5628 \\
\hline ZTIF-1 & 1430 & 1981 & 86 & 22.6 & Chem.Commun. 2014, 50, 12065-12068 \\
\hline IFMC-1 & 780 & 932 & 91.4 & 30.7 & Chem. Sci. 2012, 3, 2114-2118 \\
\hline ZMOF-1 & 1151 & 1222 & 181.22 & 31.2 & J. Am. Chem. Soc. 2012, 134, 18892-18895 \\
\hline
\end{tabular}

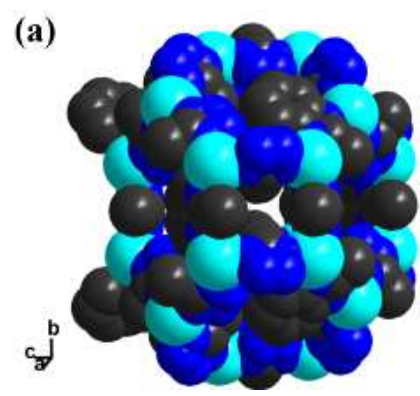

(b)

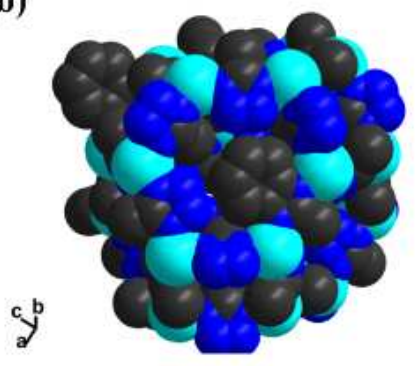

(c)

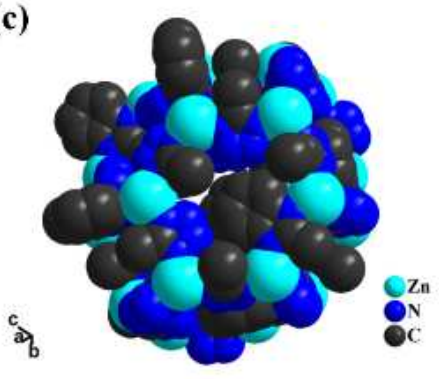

Figure S1. Space-filling modes of the SOD cage in ZTIF-6. The 4-ring window (a) and two 6-ring windows (b and c) along different directions. 
(a)

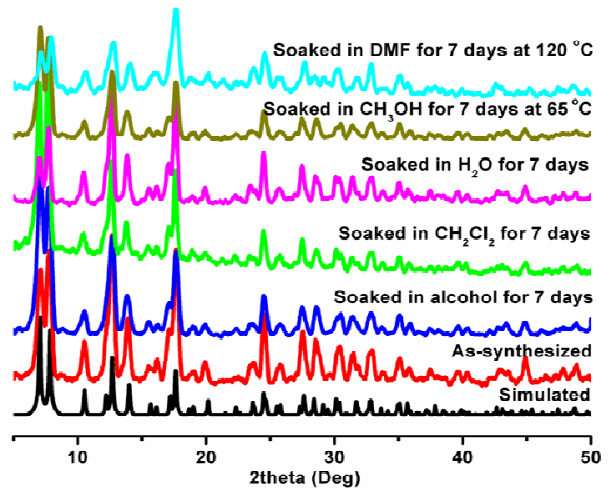

(b)

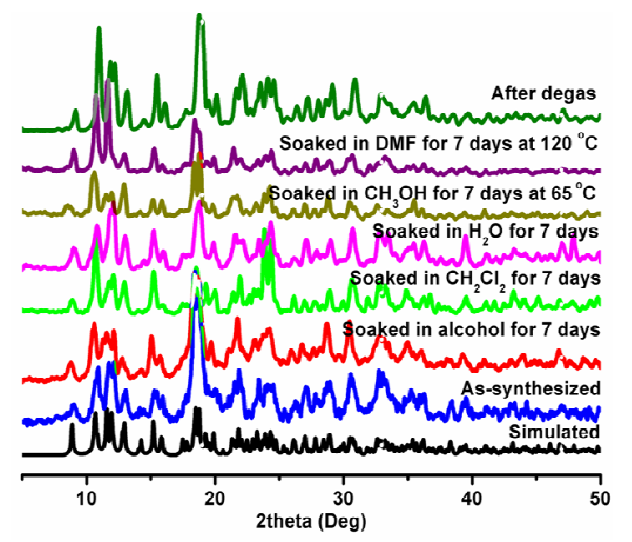

Figure S2. The powder XRD patterns of ZTIF-6 (a) and 7 (b) under different conditions.

(a)

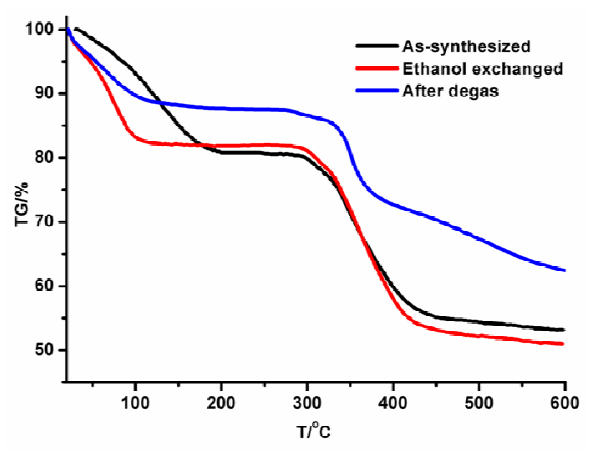

(b)

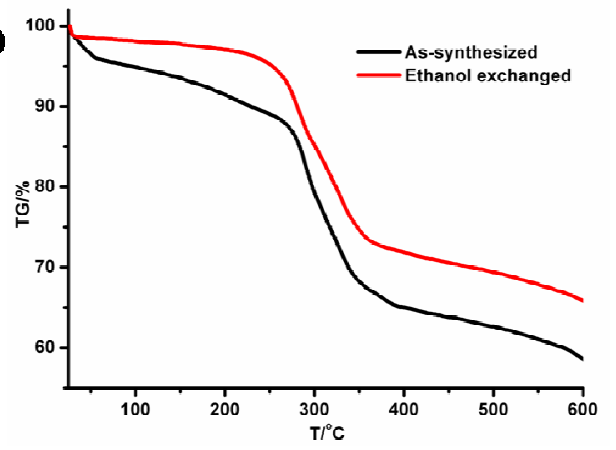

Figure S3. The TG plots of ZTIF-6 (a) and ZTIF-7 (b).

Thermogravimetric analysis (TGA) was performed to evaluate their thermal stability (Figure S3).

From the TGA plots (a), the weight loss before $200{ }^{\circ} \mathrm{C}$ was due to the guest molecules. After 270

${ }^{\circ} \mathrm{C}$, the framework decomposition started, which indicates that the network structure of ZTIF-6 is stable below that temperature. After the ethanol exchange, the platform occurs at low temperature (ca. $100{ }^{\circ} \mathrm{C}$ ), suggesting that the guest exchange process is complete. For the as-synthesized samples of ZTIF-7 (b), the decomposition temperature is around $260{ }^{\circ} \mathrm{C}$. However, there is no obvious platform is observed, even after the long-time guest exchange process. These results mean that the guest molecules are hard to remove from the channel. The $\mathrm{N}_{2}$ adsorption of them can also prove this.

\section{Isosteric heat of gas adsorption}

The isosteric heats of adsorption were calculated according to Clausius-Clapeyron equation: 


$$
\frac{\partial(\ln P)}{\partial(1 / T)}=-\frac{Q s t}{R}
$$

where $\mathbf{Q}_{\text {st }}$ is the isosteric heats of adsorption, $\mathbf{R}$ is gas constant $\left(8.314 \mathrm{~J} /\left(\mathrm{K} \mathrm{mol}^{-1}\right)\right)$. As is shown in Figure S4, the isosteric heats of $\mathrm{C}_{2} \mathrm{H}_{6}$ adsorption for ZTIF-6 are between 21.84 to $24.07 \mathrm{~kJ} / \mathrm{mol}$. The isosteric heats of $\mathrm{C}_{2} \mathrm{H}_{4}$ adsorption (Figure S5) for ZTIF-6 are between 18.52 to $19.36 \mathrm{~kJ} / \mathrm{mol}$. The isosteric heats of $\mathrm{CH}_{4}$ adsorption (Figure S6) for ZTIF-6 are between 10.26 to $11.29 \mathrm{~kJ} / \mathrm{mol}$.

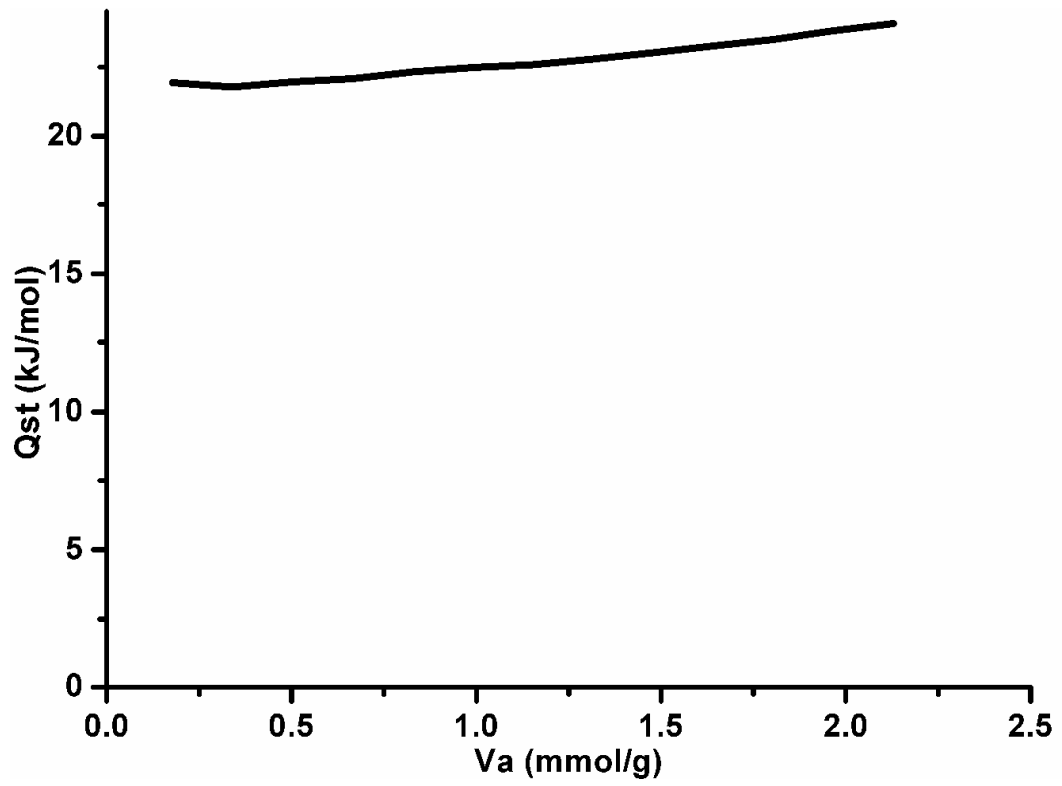

Figure S4. The isosteric heat of $\mathrm{C}_{2} \mathrm{H}_{6}$ adsorption for ZTIF-6 estimated by Clausius-Clapeyron equation. 


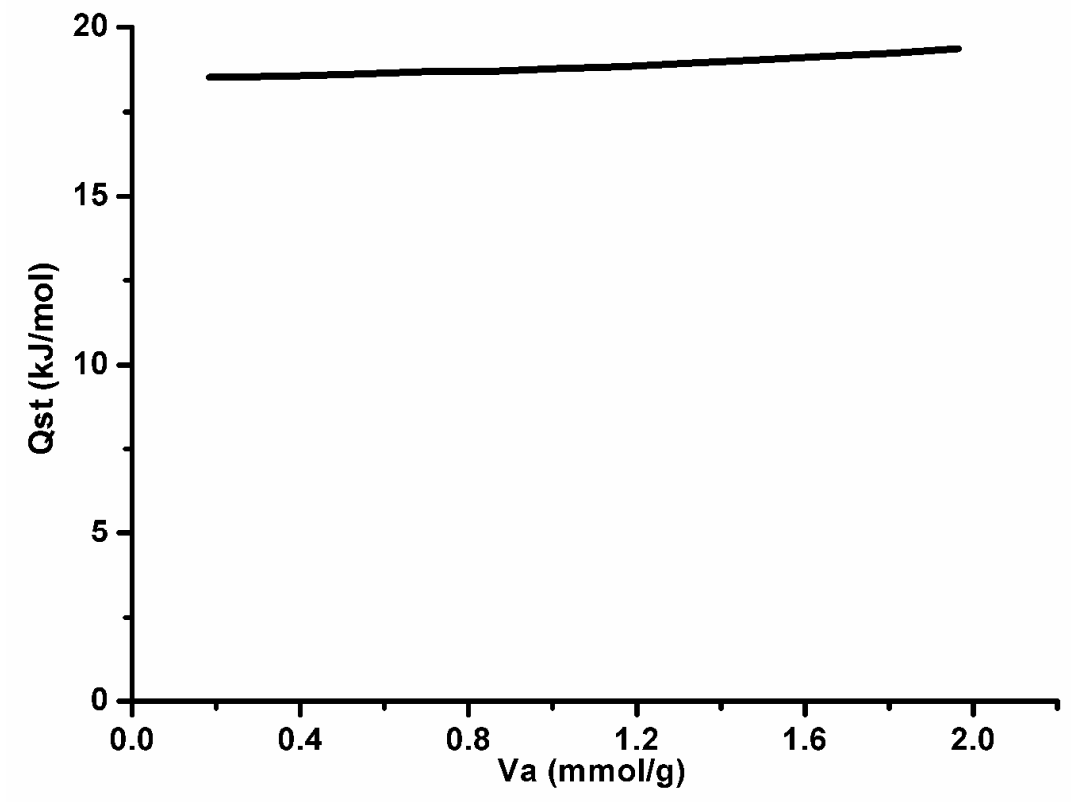

Figure S5. The isosteric heat of $\mathrm{C}_{2} \mathrm{H}_{4}$ adsorption for ZTIF-6 estimated by Clausius-Clapeyron equation.

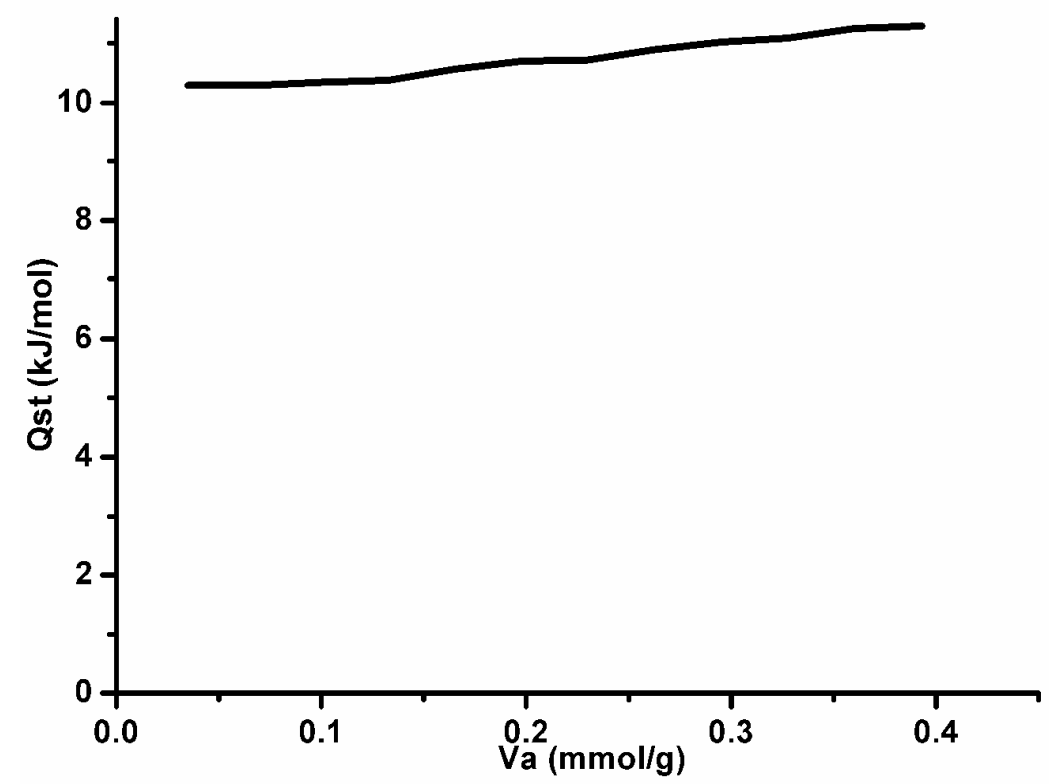

Figure S6. The isosteric heat of $\mathrm{CH}_{4}$ adsorption for ZTIF-6 estimated by Clausius-Clapeyron equation. 


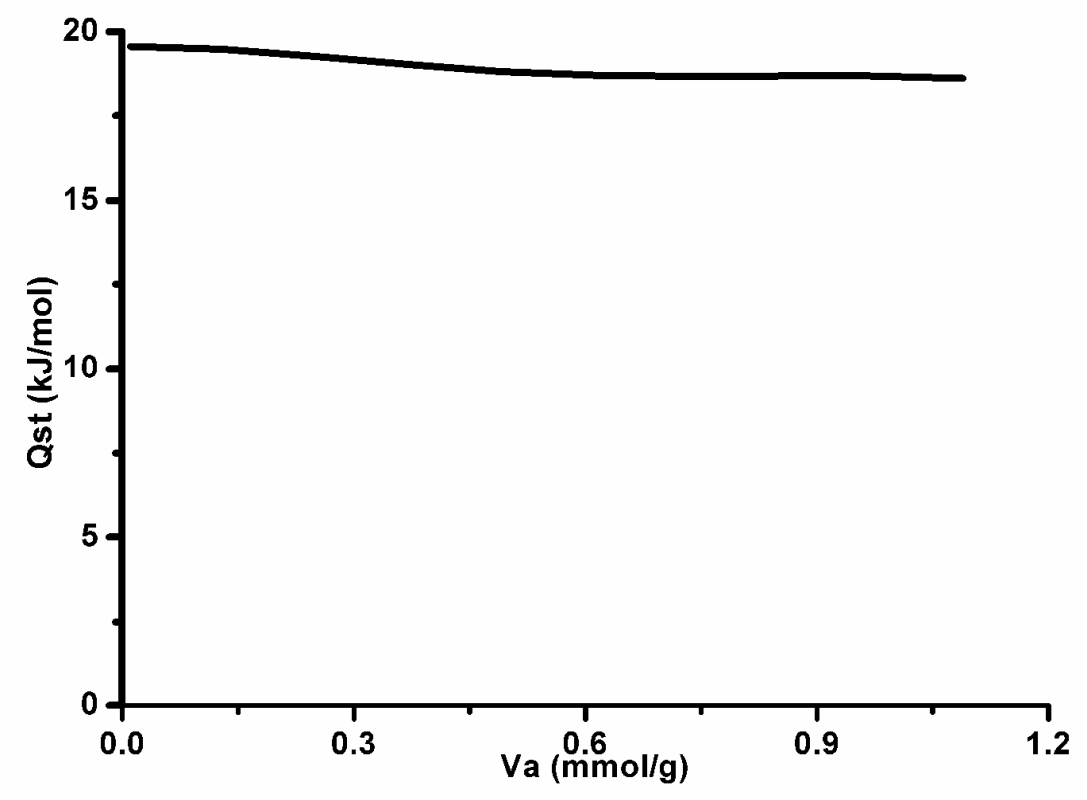

Figure S7. The isosteric heat of $\mathrm{CO}_{2}$ adsorption for ZTIF-6 estimated by Clausius-Clapeyron equation.

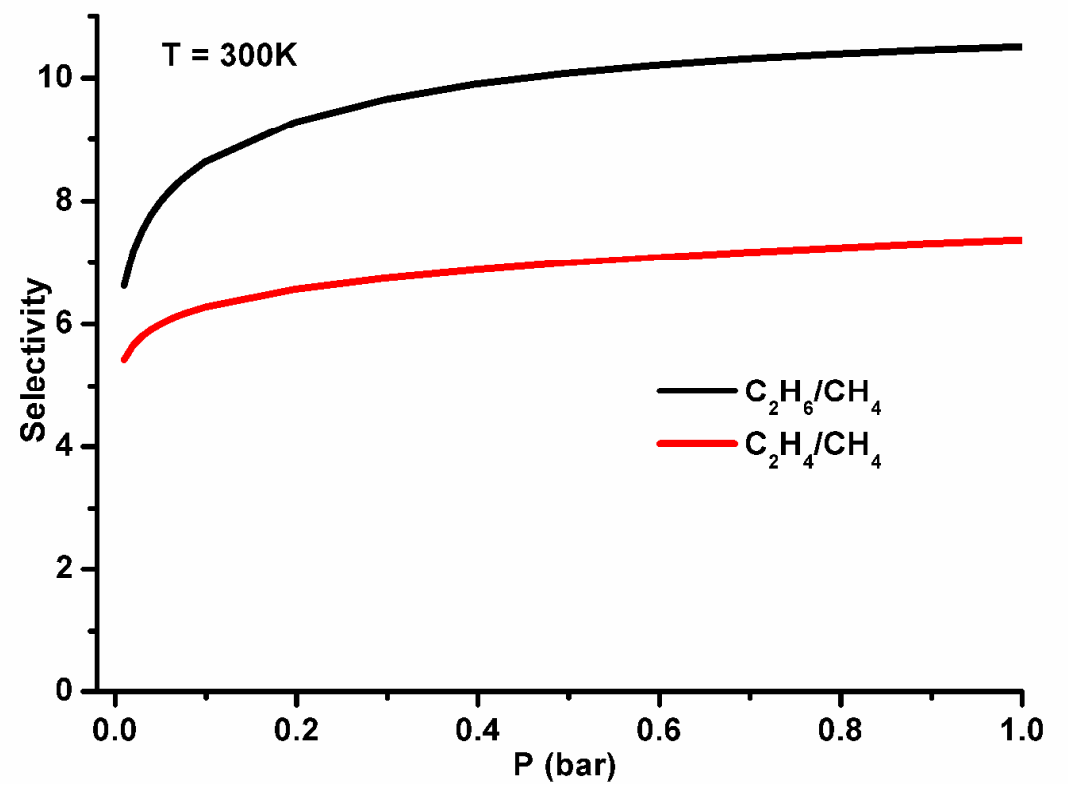

Figure S8. Mixture adsorption selectivity predicted by IAST of ZTIF-6 for $\mathrm{C}_{2} \mathrm{H}_{4}$ over $\mathrm{CH}_{4}$ (50:50) and $\mathrm{C}_{2} \mathrm{H}_{6}$ over $\mathrm{CH}_{4}(50: 50)$ dependent on pressure at $300 \mathrm{~K}$. 


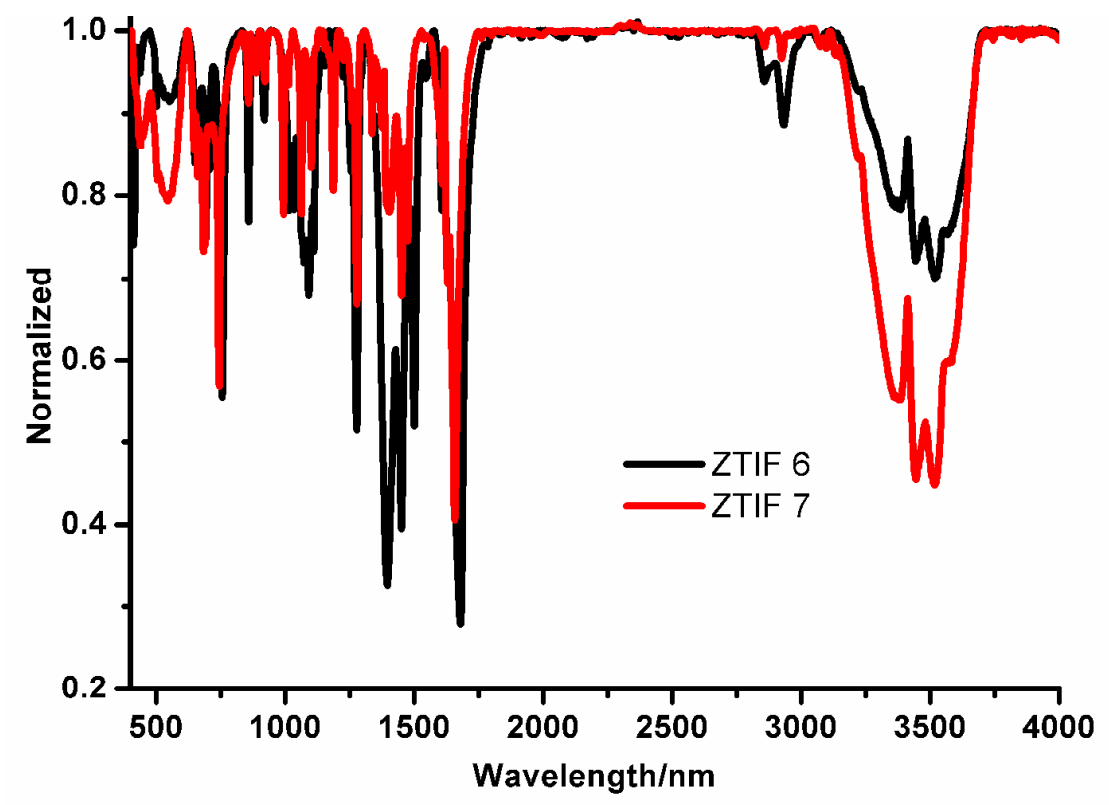

Figure S9. The FT-IR spetra of ZTIF-6 and ZTIF-7. 